Pak. j. sci. ind. res. Ser. B: biol. sci. 2019 62B(2) 77-82

\title{
In Vitro Brassica Genotypes Growth Evaluation against NaCl Salt Stress
}

\author{
Imdad Ali Mahmood*, Armghan Shahzad, Muhammad Arshad Ullah, \\ Muhammad Suhaib and Syed Ishtiaq Hyder
}

Land Resources Research Institute, National Agricultural Research Centre, Park Road, Islamabad-45500, Pakistan

(received January 31, 2017; revised September 6, 2017; accepted September 7, 2017)

\begin{abstract}
A hydroponic study was conducted to see the performance of fifteen Brassica genotypes (BARD-I, Sultan Raya, 19-H, Shiralle, Dunkled, 85-508, Toria, 85-499, BSA, 85-397, 85-500, 85-497, 85-5001, CON-I, and Jewel) for their salt tolerance. Four levels of $\mathrm{NaCl}(0,30,60$ and $90 \mathrm{mM} / \mathrm{L})$ were developed. One week old two healthy seedlings of each Brassica genotypes were transplanted in holes of thermopole sheet floating over a $200 \mathrm{~mL}$ capacity container having half strength Hoagland solution. Increasing the $\mathrm{NaCl}$ salinity significantly reduced the shoot and root dry weight of all Brassica genotypes. On an average, about $47 \%$ reduction in shoot dry weight was observed under higher salinity level ( 90 $\mathrm{mM} / \mathrm{NaCl}$ ). Although, all the genotypes differed among themselves in ability to tolerate salinity however, ranking order of genotypes showed that Sultan Raya performed well followed by BARD-I than rest of the genotypes. Genotype 85-500 produced comparatively the least shoot and root dry weights and accumulated more $\mathrm{Na}^{+}$, less $\mathrm{K}^{+}$and $\mathrm{Ca}^{+2}$ than rest of the genotypes. The reverse was true for Sultan Raya and BARD-I. The farmers could be benefitted through growing the selected comparatively more tolerant genotypes which could grow and survive under salt stress conditions rather than complete crop failure.
\end{abstract}

Keywords: Brassica genotypes, $\mathrm{NaCl}$ salt stress, hydroponic study, growth performance, germplasm screening, ion accumulation

\section{Introduction}

Brassica is one of the most important oil seed crops grown on an area of $305 \times 10^{3}$ hectares and its total oil seed production is $251 \times 10^{3}$ tonnes in Pakistan. It contributes to 21 percent of the total edible oil consumption in the country (ESP, 2015). NaCl is one of the most important salts influencing the growth of plants. Dissolved $\mathrm{Na}^{+}$and $\mathrm{Cl}^{-}$ions from $\mathrm{NaCl}$ salt cause osmotic stress in plants which interfere with root uptake of both water and nutrients (Mahmood et al., 2007; Sadiq et al., 2002). Accumulation of these ions also cause injury and toxicity in leaves resulting in reduced growth and yield (Cassaniti et al., 2009; Parida and Das, 2005). Rapeseeds and mustard are the most imperative source of edible oil (Haq et al., 2002) but the farmers do not pay much attention on this crop and grow mostly on marginal lands. The tolerance of plant to $\mathrm{NaCl}$ is generally related to the concentration of $\mathrm{Na}$ in tissues (Flowers, 2004; Ashraf and McNeilly, 2004). Besides this, the salt-affected areas can have a good potential to grow some salt tolerant genotypes of oil seed crops and hence, oil seed production can be increased by exploiting genetic potential of Brassica cultivars. Initially, salinity tolerance of Brassica germplasms was tested *Author for correspondence; E-mail: imdadlrri@gmail.com with $\mathrm{NaCl}$ salt before sowing directly in salt-affected fields because $\mathrm{NaCl}$ salt is generally considered comparatively more toxic due to its high solubility and higher uptake of $\mathrm{Na}^{+}$and $\mathrm{Cl}^{-}$ions than rest of the salts (Bilkis et al., 2016; Lee and Iersel, 2008; Chen et al., 2003) therefore, the species which could tolerate $\mathrm{NaCl}$ stress may be able to stand with other salts under field conditions. Keeping in view the fact, a hydroponic study was conducted at National Agricultural Research Centre during 2014 to see the performance of fifteen Brassica genotypes (BARD-I, Sultan Raya, 19-H, Shiralle, Dunkled, 85-508, Toria, 85-499, BSA, 85-397, 85-500, $85-497,85-5001$, CON-I and Jewel) for their salinity tolerance.

\section{Materials and Methods}

Viable seeds of each genotype were sown in polyethylene coated trays containing washed sand. Distilled water was used to maintain optimum moisture for germination and seedlings establishment. One week old two healthy seedlings of each genotype were transplanted in foam plugged thermopole sheets floating over half strength Hoagland nutrient solution (Hoagland and Arnon, 1950) of $200 \mathrm{~L}$ capacity tubs. Four levels of $\mathrm{NaCl}(0,30,60$ and $90 \mathrm{mM} / \mathrm{L}$ ) were developed in three increments after 
the complete establishment of seedlings. Aeration in the rooting medium was provided throughout growth period by air pumps. The $\mathrm{pH}$ of the solution was adjusted around 6.0 by adding $1 \mathrm{~N} \mathrm{HCl}$ or $\mathrm{NaOH}$. Arrangement of treatments was done according to completely randomize design (CRD) with five replications. After four weeks of growth, the plants of each genotype were harvested, washed with distilled water and dried by using tissue paper. Shoots and roots were separated for taking their length and fresh weights. Plant samples thus collected were dried at $60{ }^{\circ} \mathrm{C}$ to a constant weight and then weighed for their dry weights. The samples were ground to pass through $40 \mathrm{~mm}$ mesh sieve using Wiley Mills. Ground samples of root and shoot were digested in 2:1 di-acid mixture (2 Perchloric +1 Nitric acid) to estimate $\mathrm{Na}^{+}, \mathrm{K}^{+}, \mathrm{Ca}^{+2}$ and $\mathrm{Mg}^{+2}$ using atomic absorption spectroscopy. The data thus collected were subjected to statistical analysis and treatment means were compared using Duncan's Multiple Range (DMR) test (Gomez and Gomez, 1984).

\section{Results and Discussion}

Increase in $\mathrm{NaCl}$ salinity decreased shoot dry weight of all Brassica genotypes. There were non-significant

Table 1. Shoot dry weight (SDW, g/plant) of Brassica genotypes as affected by different levels of $\mathrm{NaCl}$ salinity (mean of five replications)

\begin{tabular}{llllll}
\hline \hline Variety & \multirow{2}{*}{ Control } & $30 \mathrm{mM}$ & $60 \mathrm{mM}$ & $90 \mathrm{mM}$ & \multirow{2}{*}{ Means } \\
\cline { 3 - 5 } & & \multicolumn{5}{c}{$\mathrm{NaCl}$} \\
\hline BARD-I & $4.02 \mathrm{~b} *$ & $3.87 \mathrm{~b}$ & $2.12 \mathrm{bc}$ & $2.86 \mathrm{~b}$ & $3.22 \mathrm{~B}$ \\
Sultan & $8.48 \mathrm{a}$ & $6.86 \mathrm{a}$ & $3.26 \mathrm{a}$ & $3.88 \mathrm{a}$ & $5.62 \mathrm{~A}$ \\
Raya & & & & & \\
19-H & $3.36 \mathrm{bcd}$ & $3.87 \mathrm{~b}$ & $1.41 \mathrm{de}$ & $1.49 \mathrm{~cd}$ & $2.53 \mathrm{C}$ \\
Shiralle & $1.90 \mathrm{e}$ & $1.60 \mathrm{ef}$ & $1.71 \mathrm{~cd}$ & $1.77 \mathrm{c}$ & $1.75 \mathrm{DE}$ \\
Dunkled & $2.05 \mathrm{e}$ & $0.92 \mathrm{f}$ & $1.44 \mathrm{de}$ & $1.43 \mathrm{cde}$ & $1.46 \mathrm{EF}$ \\
$85-508$ & $1.82 \mathrm{e}$ & $1.34 \mathrm{f}$ & $1.04 \mathrm{ef}$ & $0.95 \mathrm{ef}$ & $1.29 \mathrm{FG}$ \\
Toria & $3.89 \mathrm{~b}$ & $2.68 \mathrm{~cd}$ & $2.27 \mathrm{~b}$ & $1.35 \mathrm{cde}$ & $2.55 \mathrm{C}$ \\
$85-499$ & $2.16 \mathrm{e}$ & $0.94 \mathrm{f}$ & $1.42 \mathrm{de}$ & $0.69 \mathrm{f}$ & $1.30 \mathrm{FG}$ \\
BSA & $2.60 \mathrm{cde}$ & $1.84 \mathrm{def}$ & $1.85 \mathrm{bcd}$ & $1.44 \mathrm{cde}$ & $1.93 \mathrm{D}$ \\
$85-397$ & $2.70 \mathrm{cde}$ & $1.34 \mathrm{f}$ & $0.54 \mathrm{f}$ & $1.18 \mathrm{def}$ & $1.44 \mathrm{EF}$ \\
$85-500$ & $0.60 \mathrm{f}$ & $1.48 \mathrm{ef}$ & $0.91 \mathrm{ef}$ & $1.00 \mathrm{def}$ & $1.00 \mathrm{G}$ \\
$85-497$ & $2.13 \mathrm{e}$ & $1.25 \mathrm{f}$ & $0.82 \mathrm{f}$ & $1.72 \mathrm{c}$ & $1.48 \mathrm{EF}$ \\
$85-5001$ & $3.74 \mathrm{bc}$ & $2.93 \mathrm{bc}$ & $2.02 \mathrm{bc}$ & $1.16 \mathrm{def}$ & $2.46 \mathrm{C}$ \\
CON-I & $1.85 \mathrm{e}$ & $2.42 \mathrm{cde}$ & $1.69 \mathrm{~cd}$ & $1.41 \mathrm{cde}$ & $1.85 \mathrm{DE}$ \\
Jewel & $2.23 \mathrm{de}$ & $1.41 \mathrm{f}$ & $0.86 \mathrm{f}$ & $0.74 \mathrm{f}$ & $1.31 \mathrm{FG}$ \\
Mean & $2.90 \mathrm{~A}$ & $2.32 \mathrm{~B}$ & $1.56 \mathrm{C}$ & $1.54 \mathrm{C}$ & \\
\hline \hline * means bearing same letter(s) in each column are statistically \\
similar at p $\leq 0.05$. & & & &
\end{tabular}

differences between $60 \mathrm{mM} / \mathrm{L}$ and $90 \mathrm{mM} / \mathrm{L}$ salinity levels in terms of shoot dry weight in all genotypes, however, genotypes differed significantly among each other at all salinity levels (Table 1). Akhtar et al. (2002) has shown the adverse effects of salinity on Brassica growth and reported variable responses of different species and varieties of Brassica to salt stress. Root dry weight of all genotypes was also adversely affected due to increasing the level of salt stress except for Sultan Raya (Table 2). There was a comparable increase in root dry weight in case of Sultan Raya with increase in shoot dry weight. Among all genotype, Sultan Raya performed the best even under higher $\mathrm{NaCl}$ salinity ( 90 $\mathrm{mM} / \mathrm{L}$ ) and recorded as the most salt tolerant genotype whereas the genotype 85-500 was the least salt tolerant with respect to total biomass production.Sodium content (\%) in shoot and root of different Brassica genotypes increased with increase in salinity. However, there were non-significant differences between genotypes for $\mathrm{Na}^{+}$ content in shoots under control, $30 \mathrm{mM} / \mathrm{L}$ and $90 \mathrm{mM} / \mathrm{L}$ $\mathrm{NaCl}$ concentration (Table 3).Genotype Sultan Raya produced more shoot and root dry weight and accumulated less $\mathrm{Na}^{+}$in its shoot while genotype 85-500 produced least shoot and root dry weights and

Table 2. Root dry weight (g/plant) of Brassica genotypes as affected by different levels of $\mathrm{NaCl}$ salinity (Mean of five replications)

\begin{tabular}{llllll}
\hline \hline Variety & \multirow{2}{*}{ Control } & \multirow{2}{*}{$30 \mathrm{mM}$} & $60 \mathrm{mM}$ & $90 \mathrm{mM}$ & \multirow{2}{*}{ Means } \\
\cline { 3 - 5 } & & \multicolumn{5}{c}{$\mathrm{NaCl}$} \\
\hline BARD-I & $0.21 \mathrm{~cd} *$ & $0.46 \mathrm{a}$ & $0.30 \mathrm{a}$ & $0.16 \mathrm{~cd}$ & $0.28 \mathrm{~B}$ \\
Sultan & $1.24 \mathrm{a}$ & $0.52 \mathrm{a}$ & $0.21 \mathrm{bc}$ & $0.42 \mathrm{a}$ & $0.60 \mathrm{~A}$ \\
Raya & & & & & \\
$19-\mathrm{H}$ & $0.20 \mathrm{~cd}$ & $0.17 \mathrm{~cd}$ & $0.14 \mathrm{de}$ & $0.11 \mathrm{de}$ & $0.15 \mathrm{DE}$ \\
Shiralle & $0.08 \mathrm{fg}$ & $0.11 \mathrm{de}$ & $0.23 \mathrm{~b}$ & $0.11 \mathrm{de}$ & $0.13 \mathrm{DEF}$ \\
Dunkled & $0.10 \mathrm{efg}$ & $0.08 \mathrm{e}$ & $0.11 \mathrm{~d}-\mathrm{g}$ & $0.14 \mathrm{cde}$ & $0.11 \mathrm{FG}$ \\
$85-508$ & $0.09 \mathrm{efg}$ & $0.06 \mathrm{e}$ & $0.07 \mathrm{fg}$ & $0.10 \mathrm{de}$ & $0.08 \mathrm{G}$ \\
Toria & $0.24 \mathrm{bc}$ & $0.25 \mathrm{~b}$ & $0.15 \mathrm{de}$ & $0.19 \mathrm{bc}$ & $0.21 \mathrm{C}$ \\
$85-499$ & $0.11 \mathrm{~d}-\mathrm{g}$ & $0.12 \mathrm{cde}$ & $0.12 \mathrm{~d}-\mathrm{g}$ & $0.11 \mathrm{de}$ & $0.12 \mathrm{EFG}$ \\
BSA & $0.17 \mathrm{c}-\mathrm{f}$ & $0.12 \mathrm{cde}$ & $0.17 \mathrm{~cd}$ & $0.17 \mathrm{~cd}$ & $0.16 \mathrm{D}$ \\
$85-397$ & $0.18 \mathrm{cde}$ & $0.07 \mathrm{e}$ & $0.06 \mathrm{~g}$ & $0.11 \mathrm{de}$ & $0.11 \mathrm{FG}$ \\
$85-500$ & $0.05 \mathrm{~g}$ & $0.10 \mathrm{de}$ & $0.09 \mathrm{efg}$ & $0.11 \mathrm{de}$ & $0.09 \mathrm{G}$ \\
$85-497$ & $0.16 \mathrm{c}-\mathrm{f}$ & $0.11 \mathrm{de}$ & $0.13 \mathrm{def}$ & $0.14 \mathrm{cde}$ & $0.13 \mathrm{DEF}$ \\
$85-5001$ & $0.31 \mathrm{~b}$ & $0.19 \mathrm{bc}$ & $0.20 \mathrm{bc}$ & $0.23 \mathrm{~b}$ & $0.24 \mathrm{C}$ \\
CON-I & $0.12 \mathrm{~d}-\mathrm{g}$ & $0.13 \mathrm{cde}$ & $0.12 \mathrm{~d}-\mathrm{g}$ & $0.08 \mathrm{e}$ & $0.11 \mathrm{FG}$ \\
Jewel & $0.16 \mathrm{c}-\mathrm{f}$ & $0.14 \mathrm{cde}$ & $0.09 \mathrm{efg}$ & $0.13 \mathrm{cde}$ & $0.13 \mathrm{DEF}$ \\
Mean & $0.23 \mathrm{~A}$ & $0.18 \mathrm{~B}$ & $0.15 \mathrm{C}$ & $0.15 \mathrm{C}$ & \\
\hline \hline
\end{tabular}


accumulated $\mathrm{Na}^{+}$the most. This fact was exhibited by a strong negative correlation between shoot $\mathrm{Na}^{+}$content and shoot/root dry weights in all Brassica genotypes $(-0.42$ and -0.38 , respectively, (Table 11$)$. In case of $\mathrm{Na}^{+}$content in roots (Table 4) all the genotypes differed significantly at all salinity levels. Genotypes 85-500 accumulated more $\mathrm{Na}^{+}$in its roots than all other genotypes followed by Sultan Raya, 85-508, 85-397, Jewel, BSA, 85-5001, Toria, Dunkeld, 85-497, CON-1, 19-H, 85-499 and BARD-I. Genotype Sultan Raya accumulated more $\mathrm{Na}^{+}$than all other genotypes except 85-500. $\mathrm{Na}^{+}$content of root in Sultan Raya were equal to $\mathrm{Na}^{+}$ content in shoot $(1.90 \%)$ while $\mathrm{Na}^{+}$content in shoot increased significantly as compared with roots in all other genotypes (Table 4-5). The existence of genotypic variation for salt tolerance representing the physiological characters; the difficulty could be faced if the differences between genotypes are very minute considering the physiological parameters i.e. $\mathrm{K}^{+}$and $\mathrm{Ca}^{+2}$ contents (Bilkis et al., 2016; Cassaniti et al., 2009; Shirazi et al., 2007; Mahmood et al., 2007). Potassium content of shoot and root (Table 5-6) in Brassica genotypes decreased with increase in salinity. Genotype BSA contained more potassium than rest of the genotypes. All other genotypes followed the ranking order as BARD- $1>85-500>85-508>$ Sultan Raya $>85-397$ $>$ Jewel $>19-\mathrm{H}>$ Shiralle $>$ Toria $>85-499>85-5001$ $>$ CON-1 $>$ Dunkled $>85-497$. In case of root potassium content, the order was somewhat different such as Shiralle $>85-500>19-\mathrm{H}>\mathrm{CON}-1>85-397>$ Sultan Raya $>85-499>85-5001>$ BARD- $1>$ Jewel $>$ Toria $>$ BSA $>$ Dunkled $>85-508>85-497$.

It was noted that there was a poor correlation between shoot/root potassium content and shoot/root dry weights (Table 11). The fact was again depicted by higher $\mathrm{K}^{+}$ content of roots in case of 85-500 than in Sultan Raya. There was a significant positive correlation between shoot $\mathrm{K}^{+}$content and root $\mathrm{Na}^{+}$content in all genotypes (Table 11). Sodium chloride salinity significantly affected the calcium uptake by plants with increase in salinity (Table 7-8). Sultan Raya exhibited 23\% more calcium contents as compared to genotype 85-500. This indicated that high $\mathrm{Na}^{+}$concentration induced $\mathrm{Ca}^{+2}$ deficiencies in plant which effected plant growth (correlation coefficient of -0.321 , Table 11) because calcium concentration in root was also significantly affected with increase in salinity level (Table 8). Where salinity level was zero, $\mathrm{Ca}^{+2}$ uptake was high. However, increase in $\mathrm{NaCl}$ salinity level, $\mathrm{Ca}^{+2}$ uptake were reduced. Positive
Table 3. Sodium content (\%) in shoot of Brassica genotypes as affected by different levels of $\mathrm{NaCl}$ salinity (Mean of three replications)

\begin{tabular}{llllll}
\hline \hline Variety & \multirow{2}{*}{ Control } & $30 \mathrm{mM}$ & $60 \mathrm{mM}$ & $90 \mathrm{mM}$ & \multirow{2}{*}{ Means } \\
\cline { 3 - 5 } & & \multicolumn{5}{c}{$\mathrm{NaCl}$} \\
\hline BARD-I & 0.43 N.S. & 1.09 N.S. & $3.36 \mathrm{de}$ & $3.52 \mathrm{~N} . S$. & $2.10 \mathrm{ABC}$ \\
Sultan & 0.36 & 1.11 & $3.11 \mathrm{de}$ & 3.03 & $1.90 \mathrm{CD}$ \\
Raya & & & & & \\
19-H & 0.49 & 1.23 & $3.37 \mathrm{de}$ & 4.45 & $2.39 \mathrm{AB}$ \\
Shiralle & 0.40 & 1.24 & $3.36 \mathrm{de}$ & 4.57 & $2.39 \mathrm{AB}$ \\
Dunkled & 0.41 & 1.28 & $3.42 \mathrm{de}$ & 3.63 & $2.49 \mathrm{~A}$ \\
$85-508$ & 0.39 & 1.45 & $3.58 \mathrm{bcd}$ & 5.27 & $2.19 \mathrm{AB}$ \\
Toria & 0.42 & 1.99 & $4.15 \mathrm{ab}$ & 4.75 & $2.83 \mathrm{~A}$ \\
$85-499$ & 0.34 & 1.12 & $4.26 \mathrm{a}$ & 4.69 & $2.60 \mathrm{~A}$ \\
BSA & 0.43 & 1.94 & $4.01 \mathrm{abc}$ & 5.18 & $2.89 \mathrm{~A}$ \\
$85-397$ & 0.38 & 1.65 & $2.96 \mathrm{e}$ & 2.78 & $1.94 \mathrm{C}$ \\
$85-500$ & 0.32 & 1.15 & $4.43 \mathrm{a}$ & 5.02 & $2.73 \mathrm{~A}$ \\
$85-497$ & 0.36 & 1.95 & $3.46 \mathrm{cde}$ & 4.38 & $2.54 \mathrm{AB}$ \\
$85-5001$ & 0.36 & 1.94 & $3.63 \mathrm{bcd}$ & 4.47 & $2.60 \mathrm{~A}$ \\
CON-I & 0.31 & 1.91 & $2.90 \mathrm{e}$ & 3.52 & $2.16 \mathrm{ABC}$ \\
Jewel & 0.35 & 1.89 & $4.13 \mathrm{ab}$ & 3.41 & $2.45 \mathrm{AB}$ \\
Mean & $0.38 \mathrm{~B}$ & 1.49 & $3.61 \mathrm{~A}$ & $4.18 \mathrm{~A}$ & \\
\hline \hline
\end{tabular}

$*=$ means bearing same letter(s) in each column are statistically similar at $\mathrm{p} \leq 0.05$; NS $=$ means in each column are nonsignificant.

Table 4. Sodium content (\%) in root of Brassica genotypes as affected by different levels of $\mathrm{NaCl}$ salinity (Mean of three replications)

\begin{tabular}{llllll}
\hline \hline Variety & \multirow{2}{*}{ Control } & $30 \mathrm{mM}$ & $60 \mathrm{mM}$ & $90 \mathrm{mM}$ & \multirow{2}{*}{ Means } \\
\cline { 3 - 5 } & & \multicolumn{5}{c}{ NaCl } \\
\hline BARD-I & $0.45 \mathrm{c}^{*}$ & $0.87 \mathrm{~h}$ & $1.61 \mathrm{~b}-\mathrm{e}$ & $0.59 \mathrm{f}$ & $0.88 \mathrm{H}$ \\
Sultan & $0.48 \mathrm{c}$ & $1.89 \mathrm{ab}$ & $2.34 \mathrm{a}$ & $2.86 \mathrm{a}$ & $1.90 \mathrm{AB}$ \\
Raya & & & & & \\
19-H & $0.51 \mathrm{c}$ & $1.07 \mathrm{gh}$ & $1.55 \mathrm{~b}-\mathrm{f}$ & $1.21 \mathrm{e}$ & $1.09 \mathrm{GH}$ \\
Shiralle & $0.61 \mathrm{c}$ & $1.43 \mathrm{cde}$ & $1.78 \mathrm{a}-\mathrm{d}$ & $2.21 \mathrm{abc}$ & $1.51 \mathrm{CDE}$ \\
Dunkled & $0.35 \mathrm{c}$ & $1.19 \mathrm{efg}$ & $1.60 \mathrm{~b}-\mathrm{e}$ & $1.92 \mathrm{bcd}$ & $1.27 \mathrm{EFG}$ \\
$85-508$ & $1.38 \mathrm{c}$ & $1.82 \mathrm{bc}$ & $1.53 \mathrm{~b}-\mathrm{f}$ & $2.46 \mathrm{abc}$ & $1.80 \mathrm{BC}$ \\
Toria & $0.38 \mathrm{c}$ & $1.22 \mathrm{~d}-\mathrm{g}$ & $1.67 \mathrm{a}-\mathrm{d}$ & $1.91 \mathrm{bcd}$ & $1.30 \mathrm{D}-\mathrm{G}$ \\
$85-499$ & $0.58 \mathrm{c}$ & $1.10 \mathrm{gh}$ & $1.13 \mathrm{def}$ & $1.43 \mathrm{de}$ & $1.06 \mathrm{GH}$ \\
BSA & $0.43 \mathrm{c}$ & $1.39 \mathrm{def}$ & $1.98 \mathrm{abc}$ & $2.06 \mathrm{bcd}$ & $1.47 \mathrm{DEF}$ \\
$85-397$ & $0.46 \mathrm{c}$ & $1.52 \mathrm{bcd}$ & $1.90 \mathrm{abc}$ & $2.50 \mathrm{ab}$ & $1.60 \mathrm{BCD}$ \\
$85-500$ & $2.47 \mathrm{a}$ & $2.13 \mathrm{a}$ & $2.03 \mathrm{ab}$ & $1.80 \mathrm{cde}$ & $2.11 \mathrm{~A}$ \\
$85-497$ & $0.37 \mathrm{c}$ & $1.18 \mathrm{efg}$ & $0.94 \mathrm{ef}$ & $2.53 \mathrm{ab}$ & $1.26 \mathrm{EFG}$ \\
$85-5001$ & $0.49 \mathrm{c}$ & $1.34 \mathrm{def}$ & $1.33 \mathrm{c}-\mathrm{f}$ & $2.51 \mathrm{ab}$ & $1.42 \mathrm{DEF}$ \\
CON-I & $0.47 \mathrm{c}$ & $1.11 \mathrm{fg}$ & $0.89 \mathrm{f}$ & $2.25 \mathrm{abc}$ & $1.18 \mathrm{FG}$ \\
Jewel & $0.43 \mathrm{c}$ & $1.41 \mathrm{def}$ & $2.11 \mathrm{ab}$ & $2.01 \mathrm{bcd}$ & $1.49 \mathrm{DEF}$ \\
Mean & $0.67 \mathrm{C}$ & $1.44 \mathrm{~B}$ & $1.63 \mathrm{~B}$ & $2.02 \mathrm{~A}$ & \\
\hline \hline
\end{tabular}

$*=$ means bearing same letter(s) in each column are statistically similar at $\mathrm{p} \leq 0.05$. 
Table 5. Potassium content (\%) in shoot of Brassica genotypes as affected by different levels of $\mathrm{NaCl}$ salinity (Mean of three replications)

\begin{tabular}{lllllll}
\hline \hline Variety & \multirow{2}{*}{ Control } & $30 \mathrm{mM}$ & $60 \mathrm{mM}$ & $90 \mathrm{mM}$ & Means \\
\cline { 3 - 5 } & & \multicolumn{5}{c}{$\mathrm{NaCl}$} \\
\cline { 3 - 6 } BARD-I & $26.76 \mathrm{~N}$ N.S. & $14.65 \mathrm{ab} *$ & $8.00 \mathrm{ab}$ & $9.19 \mathrm{a}$ & $14.65 \mathrm{AB}$ \\
Sultan & 25.41 & $13.72 \mathrm{a}-\mathrm{d}$ & $9.26 \mathrm{a}$ & $6.49 \mathrm{~b}$ & $13.72 \mathrm{~A}-\mathrm{D}$ \\
Raya & & & & & \\
19-H & 25.46 & $13.00 \mathrm{~b}-\mathrm{f}$ & $7.53 \mathrm{abc}$ & $5.99 \mathrm{bc}$ & $13.00 \mathrm{~B}-\mathrm{F}$ \\
Shiralle & 26.51 & $12.73 \mathrm{c}-\mathrm{g}$ & $6.37 \mathrm{~b}-\mathrm{e}$ & $5.30 \mathrm{bcd}$ & $12.73 \mathrm{C}-\mathrm{G}$ \\
Dunkled & 24.69 & $11.42 \mathrm{fg}$ & $5.26 \mathrm{def}$ & $4.31 \mathrm{~cd}$ & $11.42 \mathrm{FG}$ \\
$85-508$ & 30.11 & $13.77 \mathrm{a}-\mathrm{d}$ & $6.65 \mathrm{~b}-\mathrm{e}$ & $4.53 \mathrm{~cd}$ & $13.77 \mathrm{~A}-\mathrm{D}$ \\
Toria & 28.09 & $12.72 \mathrm{c}-\mathrm{g}$ & $5.54 \mathrm{c}-\mathrm{f}$ & $4.54 \mathrm{~cd}$ & $12.72 \mathrm{C}-\mathrm{G}$ \\
$85-499$ & 26.59 & $12.68 \mathrm{c}-\mathrm{g}$ & $5.80 \mathrm{c}-\mathrm{f}$ & $5.65 \mathrm{bcd}$ & $12.68 \mathrm{C}-\mathrm{G}$ \\
BSA & 28.00 & $14.95 \mathrm{a}$ & $7.03 \mathrm{bcd}$ & $9.82 \mathrm{a}$ & $14.95 \mathrm{~A}$ \\
$85-397$ & 31.10 & $13.33 \mathrm{a}-\mathrm{e}$ & $4.75 \mathrm{ef}$ & $4.13 \mathrm{~d}$ & $13.33 \mathrm{~A}-\mathrm{E}$ \\
$85-500$ & 28.83 & $14.44 \mathrm{abc}$ & $6.25 \mathrm{~b}-\mathrm{e}$ & $8.26 \mathrm{a}$ & $14.45 \mathrm{ABC}$ \\
$85-497$ & 23.47 & $11.07 \mathrm{~g}$ & $4.70 \mathrm{ef}$ & $5.04 \mathrm{bcd}$ & $11.07 \mathrm{G}$ \\
$85-5001$ & 26.44 & $12.55 \mathrm{~d}-\mathrm{f}$ & $6.76 \mathrm{~b}-\mathrm{e}$ & $4.45 \mathrm{~cd}$ & $12.55 \mathrm{D}-\mathrm{F}$ \\
CON-I & 26.02 & $11.57 \mathrm{efg}$ & $3.93 \mathrm{f}$ & $4.77 \mathrm{~cd}$ & $11.57 \mathrm{EFG}$ \\
Jewel & 27.00 & $13.04 \mathrm{~b}-\mathrm{f}$ & $7.02 \mathrm{bcd}$ & $5.11 \mathrm{bcd}$ & $13.04 \mathrm{~B}-\mathrm{F}$ \\
Mean & $26.97 \mathrm{~A}$ & $13.04 \mathrm{~B}$ & $6.32 \mathrm{C}$ & $5.84 \mathrm{C}$ & \\
\hline \hline
\end{tabular}

* = means bearing same letter(s) in each column are statistically similar at $\mathrm{p} \leq 0.05$; NS $=$ means in each column are nonsignificant.

Table 7. Calcium content (\%) in shoot of Brassica genotypes as affected by different levels of $\mathrm{NaCl}$ salinity (Mean of three replications)

\begin{tabular}{llllll}
\hline \hline Variety & \multirow{2}{*}{ Control } & $30 \mathrm{mM}$ & $60 \mathrm{mM}$ & $90 \mathrm{mM}$ & \multirow{2}{*}{ Means } \\
\cline { 3 - 5 } & & \multicolumn{5}{c}{$\mathrm{NaCl}$} \\
\hline BARD-I & $4.49 \mathrm{~b}-\mathrm{e}^{*}$ & $4.30 \mathrm{a}$ & $3.11 \mathrm{a}$ & $2.30 \mathrm{bcd}$ & $3.55 \mathrm{BC}$ \\
Sultan & $4.43 \mathrm{cde}$ & $4.11 \mathrm{a}$ & $2.86 \mathrm{abc}$ & $3.89 \mathrm{a}$ & $3.82 \mathrm{~A}$ \\
Raya & & & & & \\
19-H & $4.56 \mathrm{~b}-\mathrm{e}$ & $4.21 \mathrm{a}$ & $2.88 \mathrm{abc}$ & $2.20 \mathrm{bcd}$ & $3.46 \mathrm{CD}$ \\
Shiralle & $4.23 \mathrm{e}$ & $3.99 \mathrm{ab}$ & $2.43 \mathrm{def}$ & $2.66 \mathrm{bc}$ & $3.33 \mathrm{CD}$ \\
Dunkled & $5.34 \mathrm{~b}$ & $4.62 \mathrm{a}$ & $2.79 \mathrm{a}-\mathrm{d}$ & $2.73 \mathrm{~b}$ & $3.87 \mathrm{AB}$ \\
$85-508$ & $5.22 \mathrm{bc}$ & $4.32 \mathrm{a}$ & $2.65 \mathrm{bcd}$ & $2.09 \mathrm{~cd}$ & $3.57 \mathrm{BC}$ \\
Toria & $4.33 \mathrm{de}$ & $3.40 \mathrm{~b}$ & $2.64 \mathrm{bcd}$ & $2.03 \mathrm{~cd}$ & $3.10 \mathrm{CD}$ \\
$85-499$ & $6.51 \mathrm{a}$ & $4.79 \mathrm{a}$ & $2.44 \mathrm{def}$ & $2.42 \mathrm{bcd}$ & $4.04 \mathrm{~A}$ \\
BSA & $4.17 \mathrm{e}$ & $3.58 \mathrm{ab}$ & $2.64 \mathrm{bcd}$ & $2.39 \mathrm{bcd}$ & $3.20 \mathrm{CD}$ \\
$85-397$ & $5.13 \mathrm{bcd}$ & $4.17 \mathrm{a}$ & $2.54 \mathrm{cde}$ & $1.83 \mathrm{~d}$ & $3.28 \mathrm{CD}$ \\
$85-500$ & $3.92 \mathrm{e}$ & $2.72 \mathrm{c}$ & $2.95 \mathrm{ab}$ & $2.50 \mathrm{bc}$ & $3.02 \mathrm{CD}$ \\
$85-497$ & $4.05 \mathrm{e}$ & $3.24 \mathrm{abc}$ & $2.58 \mathrm{bcd}$ & $2.49 \mathrm{bc}$ & $3.09 \mathrm{CD}$ \\
$85-5001$ & $4.41 \mathrm{cde}$ & $3.78 \mathrm{a}$ & $2.18 \mathrm{ef}$ & $2.53 \mathrm{bc}$ & $3.23 \mathrm{CD}$ \\
CON-I & $4.11 \mathrm{e}$ & $3.87 \mathrm{a}$ & $2.45 \mathrm{def}$ & $2.05 \mathrm{~cd}$ & $3.12 \mathrm{CD}$ \\
Jewel & $4.69 \mathrm{~b}-\mathrm{e}$ & $3.99 \mathrm{a}$ & $2.12 \mathrm{f}$ & $2.15 \mathrm{bcd}$ & $3.24 \mathrm{CD}$ \\
Mean & $4.64 \mathrm{~A}$ & $3.94 \mathrm{~A}$ & $2.62 \mathrm{~B}$ & $2.42 \mathrm{C}$ & \\
\hline
\end{tabular}

$*=$ means bearing same letter(s) in each column are statistically similar at $\mathrm{p} \leq 0.05$.
Table 6. Potassium content (\%) in root of Brassica genotypes as affected by different levels of $\mathrm{NaCl}$ salinity (Mean of three replications)

\begin{tabular}{llllll}
\hline \hline Variety & \multirow{2}{*}{ Control } & $30 \mathrm{mM}$ & $60 \mathrm{mM}$ & $90 \mathrm{mM}$ & \multirow{2}{*}{ Means } \\
\cline { 3 - 5 } & & \multicolumn{5}{c}{$\mathrm{NaCl}$} \\
\hline BARD-I & $15.04 \mathrm{~cd} *$ & $8.55 \mathrm{~cd}$ & $3.71 \mathrm{ab}$ & $1.21 \mathrm{~g}$ & $7.13 \mathrm{CD}$ \\
Sultan & $16.21 \mathrm{~cd}$ & $9.46 \mathrm{bc}$ & $3.25 \mathrm{bcd}$ & $3.20 \mathrm{bc}$ & $8.03 \mathrm{BC}$ \\
Raya & & & & & \\
$19-\mathrm{H}$ & $16.72 \mathrm{~cd}$ & $9.92 \mathrm{bc}$ & $4.31 \mathrm{ab}$ & $3.02 \mathrm{bcd}$ & $8.49 \mathrm{BC}$ \\
Shiralle & $22.12 \mathrm{~b}$ & $10.61 \mathrm{a}$ & $2.22 \mathrm{de}$ & $1.76 \mathrm{fg}$ & $9.18 \mathrm{~A}$ \\
Dunkled & $3.72 \mathrm{ef}$ & $5.88 \mathrm{fg}$ & $3.97 \mathrm{ab}$ & $4.26 \mathrm{a}$ & $4.46 \mathrm{FG}$ \\
$85-508$ & $5.12 \mathrm{ef}$ & $5.51 \mathrm{fg}$ & $2.51 \mathrm{cde}$ & $3.19 \mathrm{bc}$ & $4.08 \mathrm{FG}$ \\
Toria & $7.76 \mathrm{e}$ & $6.61 \mathrm{ef}$ & $3.61 \mathrm{~b}$ & $2.75 \mathrm{cde}$ & $5.18 \mathrm{EF}$ \\
$85-499$ & $17.56 \mathrm{~cd}$ & $9.19 \mathrm{bc}$ & $2.10 \mathrm{e}$ & $2.21 \mathrm{def}$ & $7.77 \mathrm{BCD}$ \\
BSA & $6.14 \mathrm{e}$ & $6.42 \mathrm{f}$ & $4.79 \mathrm{a}$ & $2.62 \mathrm{cde}$ & $4.99 \mathrm{~F}$ \\
$85-397$ & $14.19 \mathrm{~d}$ & $9.45 \mathrm{~b}$ & $4.80 \mathrm{a}$ & $3.65 \mathrm{ab}$ & $8.02 \mathrm{BC}$ \\
$85-500$ & $18.95 \mathrm{a}$ & $10.59 \mathrm{a}$ & $3.94 \mathrm{ab}$ & $3.19 \mathrm{bc}$ & $9.17 \mathrm{~A}$ \\
$85-497$ & $1.72 \mathrm{f}$ & $4.58 \mathrm{~g}$ & $3.53 \mathrm{bc}$ & $2.79 \mathrm{~b}-\mathrm{e}$ & $3.16 \mathrm{G}$ \\
$85-5001$ & $17.38 \mathrm{~cd}$ & $9.19 \mathrm{bc}$ & $2.35 \mathrm{de}$ & $2.13 \mathrm{ef}$ & $7.76 \mathrm{BCD}$ \\
CON-I & $18.76 \mathrm{bc}$ & $9.49 \mathrm{~b}$ & $1.54 \mathrm{e}$ & $2.47 \mathrm{c}-\mathrm{f}$ & $8.07 \mathrm{BC}$ \\
Jewel & $13.79 \mathrm{~d}$ & $7.90 \mathrm{de}$ & $2.14 \mathrm{de}$ & $2.09 \mathrm{ef}$ & $6.48 \mathrm{DE}$ \\
Mean & $13.01 \mathrm{~A}$ & $8.22 \mathrm{~B}$ & $3.25 \mathrm{C}$ & $2.70 \mathrm{CD}$ & \\
\hline * = means bearing same letter(s) in each column are statistically \\
similar at p $\leq 0.05$. & & & & \\
\hline
\end{tabular}

Table 8. Calcium content (\%) in root of Brassica genotypes as affected by different levels of $\mathrm{NaCl}$ salinity (Mean of three replications)

\begin{tabular}{llllll}
\hline \hline Variety & \multirow{2}{*}{ Control } & \multirow{2}{*}{$30 \mathrm{mM}$} & $60 \mathrm{mM}$ & $90 \mathrm{mM}$ & \multirow{2}{*}{ Means } \\
\cline { 3 - 5 } & & \multicolumn{4}{c}{$\mathrm{NaCl}$} \\
\hline BARD-I & $1.81 \mathrm{~cd} *$ & $1.39 \mathrm{~cd}$ & $1.36 \mathrm{~cd}$ & $1.20 \mathrm{bcd}$ & $1.44 \mathrm{C}-\mathrm{F}$ \\
Sultan & $1.85 \mathrm{~cd}$ & $0.99 \mathrm{ef}$ & $1.00 \mathrm{ef}$ & $1.34 \mathrm{bcd}$ & $1.30 \mathrm{C}-\mathrm{F}$ \\
Raya & & & & & \\
$19-\mathrm{H}$ & $1.81 \mathrm{~cd}$ & $0.84 \mathrm{fg}$ & $0.90 \mathrm{efg}$ & $1.29 \mathrm{bcd}$ & $1.21 \mathrm{DEF}$ \\
Shiralle & $2.86 \mathrm{~b}$ & $1.33 \mathrm{~cd}$ & $1.11 \mathrm{def}$ & $1.05 \mathrm{~cd}$ & $1.59 \mathrm{CD}$ \\
Dunkled & $1.71 \mathrm{~cd}$ & $0.90 \mathrm{efg}$ & $0.93 \mathrm{efg}$ & $1.10 \mathrm{bcd}$ & $1.16 \mathrm{EF}$ \\
$85-508$ & $1.88 \mathrm{~cd}$ & $0.88 \mathrm{efg}$ & $1.05 \mathrm{ef}$ & $1.24 \mathrm{bcd}$ & $1.26 \mathrm{DEF}$ \\
Toria & $1.51 \mathrm{~d}$ & $0.66 \mathrm{~g}$ & $0.68 \mathrm{~g}$ & $1.31 \mathrm{bcd}$ & $1.04 \mathrm{~F}$ \\
$85-499$ & $2.74 \mathrm{bc}$ & $2.86 \mathrm{a}$ & $2.34 \mathrm{a}$ & $1.58 \mathrm{abc}$ & $2.38 \mathrm{~B}$ \\
BSA & $1.92 \mathrm{bcd}$ & $0.79 \mathrm{fg}$ & $0.82 \mathrm{fg}$ & $1.15 \mathrm{bcd}$ & $1.17 \mathrm{DEF}$ \\
$85-397$ & $1.77 \mathrm{~cd}$ & $1.87 \mathrm{~b}$ & $1.90 \mathrm{~b}$ & $1.42 \mathrm{bcd}$ & $1.74 \mathrm{CD}$ \\
$85-500$ & $6.94 \mathrm{a}$ & $3.88 \mathrm{a}$ & $0.88 \mathrm{efg}$ & $0.84 \mathrm{~d}$ & $3.14 \mathrm{~A}$ \\
$85-497$ & $1.80 \mathrm{~cd}$ & $1.62 \mathrm{c}$ & $1.61 \mathrm{c}$ & $1.44 \mathrm{bc}$ & $1.62 \mathrm{CDE}$ \\
$85-5001$ & $1.84 \mathrm{bcd}$ & $0.86 \mathrm{efg}$ & $0.92 \mathrm{efg}$ & $1.43 \mathrm{bc}$ & $1.26 \mathrm{DEF}$ \\
CON-I & $1.71 \mathrm{~cd}$ & $1.29 \mathrm{~cd}$ & $1.13 \mathrm{de}$ & $1.70 \mathrm{ab}$ & $1.46 \mathrm{C}-\mathrm{F}$ \\
Jewel & $1.85 \mathrm{bcd}$ & $1.01 \mathrm{ef}$ & $1.44 \mathrm{c}$ & $2.10 \mathrm{a}$ & $1.60 \mathrm{C}$ \\
Mean & $2.27 \mathrm{~A}$ & $1.41 \mathrm{~B}$ & $1.20 \mathrm{~B}$ & $1.35 \mathrm{~B}$ & \\
\hline \hline
\end{tabular}


Table 9. Magnesium content (\%) in shoot of Brassica genotypes as affected by different levels of $\mathrm{NaCl}$ salinity (Mean of three replications)

\begin{tabular}{llllll}
\hline \hline Variety & \multirow{2}{*}{ Control } & $30 \mathrm{mM}$ & $60 \mathrm{mM}$ & $90 \mathrm{mM}$ & \multirow{2}{*}{ Means } \\
\cline { 3 - 5 } & & \multicolumn{3}{c}{$\mathrm{NaCl}$} \\
\hline BARD-I & 0.44 & $2.61 \mathrm{~N} . S$. & $3.61 \mathrm{~N} . S$. & 3.47 N.S. & 2.53 N.S. \\
Sultan & 0.45 & 2.57 & 3.57 & 3.59 & 2.55 \\
Raya & & & & & \\
19-H & 0.49 & 2.52 & 3.52 & 3.55 & 2.52 \\
Shiralle & 0.51 & 2.58 & 3.58 & 3.55 & 2.56 \\
Dunkled & 0.52 & 2.58 & 3.58 & 3.53 & 2.55 \\
$85-508$ & 0.43 & 2.59 & 3.59 & 3.49 & 2.53 \\
Toria & 0.44 & 2.56 & 3.56 & 3.52 & 2.52 \\
$85-499$ & 0.48 & 2.58 & 3.58 & 3.54 & 2.55 \\
BSA & 0.40 & 2.54 & 3.54 & 3.54 & 2.51 \\
$85-397$ & 0.51 & 2.45 & 3.45 & 3.43 & 2.46 \\
$85-500$ & 0.44 & 2.59 & 3.59 & 3.51 & 2.53 \\
$85-497$ & 0.42 & 2.57 & 3.57 & 3.50 & 2.52 \\
$85-5001$ & 0.49 & 2.49 & 3.49 & 3.52 & 2.50 \\
CON-I & 0.39 & 2.55 & 3.55 & 3.49 & 2.50 \\
Jewel & 0.46 & 2.52 & 3.52 & 3.53 & 2.51 \\
Mean & $0.46 \mathrm{C}$ & $2.55 \mathrm{~B}$ & $3.55 \mathrm{~A}$ & $3.52 \mathrm{~A}$ & \\
\hline \hline
\end{tabular}

* = means bearing same letter(s) in each column are statistically similar at $\mathrm{p} \leq 0.05 ;$ N.S. $=$ means in each column are nonsignificant.
Table 10. Magnesium content (\%) in root of Brassica genotypes as affected by different levels of $\mathrm{NaCl}$ salinity (Mean of three replications)

\begin{tabular}{llllll}
\hline \hline Variety & \multirow{2}{*}{ Control } & $30 \mathrm{mM}$ & $60 \mathrm{mM}$ & $90 \mathrm{mM}$ & \multirow{2}{*}{ Means } \\
\cline { 3 - 5 } & & \multicolumn{4}{c}{$\mathrm{NaCl}$} \\
\hline BARD-I & $0.49 \mathrm{~b} *$ & $1.45 \mathrm{~b}$ & $3.45 \mathrm{~d}$ & $3.43 \mathrm{~N} . S$ & $2.21 \mathrm{D}$ \\
Sultan & $0.54 \mathrm{~b}$ & $1.48 \mathrm{~b}$ & $3.43 \mathrm{~d}$ & 3.48 & $2.23 \mathrm{D}$ \\
Raya & & & & & \\
19-H & $0.49 \mathrm{~b}$ & $1.48 \mathrm{~b}$ & $3.38 \mathrm{~d}$ & 3.58 & $2.23 \mathrm{D}$ \\
Shiralle & $0.87 \mathrm{~b}$ & $1.64 \mathrm{ab}$ & $3.45 \mathrm{~d}$ & 3.59 & $2.39 \mathrm{D}$ \\
Dunkled & $0.40 \mathrm{~b}$ & $1.54 \mathrm{ab}$ & $3.79 \mathrm{~cd}$ & 3.45 & $2.30 \mathrm{D}$ \\
$85-508$ & $0.53 \mathrm{~b}$ & $2.08 \mathrm{a}$ & $5.22 \mathrm{a}$ & 3.80 & $2.91 \mathrm{~A}$ \\
Toria & $0.46 \mathrm{~b}$ & $1.47 \mathrm{~b}$ & $3.43 \mathrm{~d}$ & 3.51 & $2.22 \mathrm{D}$ \\
$85-499$ & $0.57 \mathrm{~b}$ & $1.65 \mathrm{ab}$ & $3.94 \mathrm{c}$ & 3.45 & $2.40 \mathrm{D}$ \\
BSA & $0.58 \mathrm{~b}$ & $1.47 \mathrm{~b}$ & $3.41 \mathrm{~d}$ & 3.44 & $2.23 \mathrm{D}$ \\
$85-397$ & $0.50 \mathrm{~b}$ & $1.50 \mathrm{~b}$ & $3.58 \mathrm{~cd}$ & 3.41 & $2.25 \mathrm{D}$ \\
$85-500$ & $2.08 \mathrm{a}$ & $2.65 \mathrm{a}$ & $3.49 \mathrm{~d}$ & 3.69 & $2.98 \mathrm{~A}$ \\
$85-497$ & $0.56 \mathrm{~b}$ & $1.55 \mathrm{~b}$ & $3.54 \mathrm{~d}$ & 3.54 & $2.30 \mathrm{D}$ \\
$85-5001$ & $0.59 \mathrm{~b}$ & $1.51 \mathrm{~b}$ & $3.42 \mathrm{~d}$ & 3.51 & $2.26 \mathrm{D}$ \\
CON-I & $0.59 \mathrm{~b}$ & $1.90 \mathrm{a}$ & $3.73 \mathrm{~cd}$ & 4.07 & $2.58 \mathrm{C}$ \\
Jewel & $0.59 \mathrm{~b}$ & $1.98 \mathrm{a}$ & $4.59 \mathrm{~b}$ & 3.77 & $2.73 \mathrm{BC}$ \\
Mean & $0.68 \mathrm{C}$ & $1.64 \mathrm{~B}$ & $3.82 \mathrm{~A}$ & $3.60 \mathrm{~A}$ & \\
\hline \hline
\end{tabular}

* = means bearing same letter(s) in each column are statistically similar at $\mathrm{p} \leq 0.05 ;$ N.S. $=$ means in each column are nonsignificant.

Table 11. Correlation coefficients for different growth and ionic content variables recorded

\begin{tabular}{|c|c|c|c|c|c|c|c|c|}
\hline $\mathrm{Ca}^{++}$root & 1 & & & & & & & \\
\hline $\mathrm{Ca}^{++}$shoot & -0.083 & 1 & & & & & & \\
\hline $\mathrm{K}^{+}$root & $0.439 * *$ & 0.002 & 1 & & & & & \\
\hline $\mathrm{K}^{+}$shoot & 0.187 & -0.014 & 0.243 & 1 & & & & \\
\hline $\mathrm{Na}^{+}$root & $0.326^{*}$ & -0.224 & 0.13 & $0.357^{*}$ & 1 & & & \\
\hline $\mathrm{Na}^{+}$shoot & 0.154 & $-0.325^{*}$ & -0.266 & -0.017 & -0.067 & 1 & & \\
\hline RDW & $-0.284^{*}$ & $0.306^{*}$ & 0.174 & 0.24 & 0.151 & $-0.382 * *$ & 1 & \\
\hline \multirow[t]{2}{*}{ SDW } & $-0.4 * *$ & $0.288^{*}$ & 0.198 & 0.227 & 0.036 & $-0.423 * *$ & $0.97 * * *$ & 1 \\
\hline & $\mathrm{Ca}^{+2}$ root & $\mathrm{Ca}^{+2}$ shoot & $\mathrm{K}^{+}$root & $\mathrm{K}^{+}$shoot & $\mathrm{Na}^{+}$root & $\mathrm{Na}^{+}$shoot & RDW & SDW \\
\hline
\end{tabular}

$*=$ significant at $0.05 ; * *=$ significant at $0.01 ; * * *$ significant at 0.001 according to Little and Hills (1992).

correlation between shoot $\mathrm{Ca}^{+2}$ contents and shoot/root dry weights $(0.308$ and 0.288$)$ indicated positive effect of $\mathrm{Ca}^{+2}$ content on growth (Table 11). In case of root $\mathrm{Ca}^{+2}$ contents, reverse trend was observed. Genotype 85-500 accumulated more $\mathrm{Ca}^{+2}$ in roots than any other genotype and Sultan Raya accumulated far less $\mathrm{Ca}^{+2}$ than this genotype. The $\mathrm{Mg}^{+2}$ content in shoot tissues of Brassica genotypes were found to be non-significant (Table 9-10) however, a close observation indicates that increasing $\mathrm{NaCl}$ salinity in rooting medium increased tissue $\mathrm{Mg}^{+2}$ concentration and maximum content $(3.59 \%)$ were observed in the shoots of Sultan Raya under 90 $\mathrm{mM} / \mathrm{L} \mathrm{NaCl}$ salt stress. These results could be supported by the findings of Flowers (2004) and Sadiq et al. (2002).

\section{Conclusion}

Growth of all Brassica genotypes were significantly reduced with increase in $\mathrm{NaCl}$ salinity levels. Among all the genotypes, some species showed tolerance to 
salinity and performed well even under higher salinity $(90 \mathrm{mM} / \mathrm{L} \mathrm{NaCl})$ level. Among all the genotypes, Sultan Raya gave promised results and performed well even at $90 \mathrm{mM} / \mathrm{L} \mathrm{NaCl}$ salt stress while CV. 85-500 was found to be the most sensitive Brassica genotype. Performance of Sultan Raya will be tested under naturally salt-affected soil conditions.

Conflict of interest. The authors declare no conflict of interest.

\section{References}

Akhtar, J., Haq, T., Saqib, M., Mahmood, K. 2002. Effect of salinity on yield, growth and oil contents of four Brassica species. Pakistan Journal of Agricultural Sciences, 39: 76-79.

ESP, 2015. Economic Survey of Pakistan. Finance Division, Government of Pakistan, Economic Adviser's Wing, Islamabad, Pakistan.

Ashraf, M., McNeilly, T. 2004. Salinity tolerance in brassica oilseeds. Critical Reviews in Plant Sciences, 23: 157-174.

Bilkis, A., Islam, M.R., Hafiz, M.H.R., Hasan, M.A. 2016. Effect of $\mathrm{NaCl}$ induced salinity on some physiological and agronomic traits of wheat. Pakistan Journal of Botany, 48: 455-460.

Cassaniti, C., Leonardi, C., Flowers, T.J. 2009. The effects of sodium chloride on ornamental shrubs. Scientia Horticulturae, 122: 586-593.

Chen, S., Li, J., Wang, S., Fritz, E., Hutterman, A., Altman, A. 2003. Effects of $\mathrm{NaCl}$ on shoot growth, transpiration, ion compartmentation, and transport in regenerated plants of Populus euphratica and Populus tomentosa. Canadian Journal of Forestry Research, 33: 967-975.

Flowers, T.J. 2004. Improving crop salt tolerance.
Journal of Experimental Botany, 55: 307-319.

Gomez, K.A., Gomez, A.A. 1984. Statistical Procedures for Agriculture Research. $2^{\text {nd }}$ edition, John Wiley and Sons, New York, USA.

Haq, T.U., Akhtar, J., Haq, A.U., Hussain, M. 2002. Effect of soil salinity on the concentration of $\mathrm{Na}$, $\mathrm{K}$ and $\mathrm{Cl}$ in the leaf sap of four Brassica species. International Journal of Agriculture and Biology, 4: 385-388.

Hoagland, D.R., Arnon, D.I. 1950. The Water Culture Method of Growing Plants without Soil. Circular 347, College of Agriculture, University of California. Barkeley, USA.

Lee, M.K., vanIersel, M.W. 2008. Sodium chloride effects on growth, morphology, and physiology of (Chrysanthemum morifolium). Hortscience, 43: 1888-1891.

Little, T.M., Hills, F.J. 1978. Agricultural Experimentation: Design and Analysis, 368 pp., John Wiley and Sons, New York, USA.

Mahmood, I.A., Ali, A. Shahzad, A., Salim, M., Jamil, M., Akhtar. J. 2007. Yield and quality of Brassica cultivars as affected by soil salinity. Pakistan Journal of Scientific and Industrial Research, 50: 133-137.

Parida, A.K., Das, A.B. 2005. Salt tolerance and salinity effects on plants: a review. Ecotoxicology and Environmental Safety, 60: 324-349.

Sadiq, M., Jamil, M., Mehdi, S.M., Sarfraz, M., Hassan, G. 2002. Comparative performance of Brassica varieties/lines under saline sodic condition. Asian Journal of Plant Science, 1: 77-78.

Shirazi, M.U., Khan, M.A., Khanzada, B., Mujtaba, S.M., Ali, M., Mumtaz, S., Shereen, A., Siddique, A., Shah, J.A. 2007. Salt tolerance studies in some mutants of Brassica (Brassica juncea, cv: S-9). Pakistan Journal of Botany, 39: 2495-2500. 